\title{
Analysis of simple reaction time to a sinusoidal grating by means of a linear filter model of the detection process
}

\author{
YOSHIMICHI EJIMA and YOSHIO OHTANI \\ Kyoto University, Kyoto, Japan
}

\begin{abstract}
Simple reaction time (RT) to a sinusoidal grating was analyzed in terms of a linear filter model of the detection process. First, RT contrast functions were determined over a wide range of spatial frequencies and retinal illuminances. Second, calculating the time course of the linear filter's response, theoretical visual latency contrast functions were derived for the same conditions of spatial frequency and retinal illuminance as those in the RT measurements. Comparison of the two functions showed that the contrast dependence of the RT functions was much larger than that of the visual latency functions. The discrepancy between the two functions was satisfactorily described as a power function of the slope of the filter's response at threshold level. On the basis of these results, we propose a model of the RT process. According to the model, the RT process is mediated by a cascade that consists of a level detector, which includes a linear filter followed by a threshold device, and a differentiator of the filter's response.
\end{abstract}

Simple reaction time ( $R T)$, defined as the interval between stimulus onset and response initiation, has been a useful tool for psychophysical investigation of the human sensory decision process. Suprathreshold temporal properties of the visual system have been examined with measurement of RT as a function of stimulus parameters such as intensity (Bartlett \& MacLeod, 1954; Mansfield, 1973; Roufs, 1963, 1974; Vaughan, Costa, \& Gilden, 1966), exposure duration (Bruder \& Kietzman, 1973; Grossberg, 1968; Kietzman \& Gillam, 1972; Mansfield, 1973; Raab \& Fehrer, 1962; Ueno, 1976, 1977), size (Mansfield, 1973; Ueno, 1978), retinal location (Bartlett \& Sticht, 1968; Rains, 1963), wavelength (Bartlett \& Sticht, 1968; Nissen \& Pokorny, 1977; Pollack, 1968; Ueno, Pokorny, \& Smith, 1985), and adaptation level (Bartlett \& MacLeod, 1954; Burkhardt, Gottesman, \& Keenan, 1987).

If one assumes that the delay in the decision and motor process in a simple reaction task is constant for different stimuli, then the variation of RT with stimulus parameters may be identified with that of the sensory processing delay. This may be expressed mathematically as

$$
\mathrm{RT}=\min \left[t ; R(t) \geq d_{c}\right]+K,
$$

where $R(t)$ stands for the time course of the sensory signal, $d_{c}$ for the detection threshold, and $K$ for an irreducible minimum component of RT. This equation means that the sensory signal exceeds the detection threshold at the

\footnotetext{
This work was supported in part by Grants in Aid for Scientific Research from the Ministry of Education, Nos. 63510056 and 62790025 , and also by the Yamamura-Tamura Foundation. The second author is supported by a fellowship from the Japan Society for the Promotion of Science for Japanese Junior Scientists.
}

time of $t_{\min }=\min \left[t ; R(t) \geq d_{c}\right]$ (i.e., visual latency) and that the reaction occurs at $t_{\min }+K$ so that $\mathrm{RT}$ is an index of visual latency.

However, there are some indications that RT does not always serve as an accurate index of the sensory processing delay. Roufs (1974) showed that, as stimulus intensity was decreased, RT to a circular field increased more rapidly than did the visual latency obtained from simultaneity judgment data. Brauner and Lit (1976) and Williams and Lit (1983) found a similar divergence between the RT function and the visual latency function, which was derived from the Pulfrich and the Hess effects. Ejima and Ohtani (1987) showed that, as stimulus contrast was decreased, RT to a sinusoidal grating increased more quickly than did the perceptual .integration time, which was estimated with the empirical threshold duration function. These results suggest that RT may include an intensity- or contrast-dependent component other than the visual latency, thus requiring a modification of Equation 1. The present study was designed to explore such a modification, and thereby to improve our understanding of the RT process.

The approach taken here was (1) to measure RT to sinusoidal gratings as a function of contrast, (2) to estimate the visual latency as a function of contrast for the same stimuli as those in the RT measurements, (3) to quantify the discrepancy between the two measures, and (4) to explore a model of the RT process that might be used to explain the discrepancy. The second of these steps was carried out according to a linear filter model of the detection process that has been notably successful for predicting the temporal properties of the visual system at threshold levels (Bergen \& Wilson, 1985; Gorea \& Tyler, 1986; Watson, 1979, 1986; Watson \& Nachmias, 1977). The linear filter model also aided in the fourth step, 
in which the functional relation between RT and the time course of the filter's response was analyzed.

\section{MEASUREMENT OF THE RT CONTRAST FUNCTION}

\section{Method}

Stimulus and Apparatus. The stimulus was a vertical sinusoidal grating, electronically generated on a television monitor at a frame frequency of $60 \mathrm{~Hz}(16.7 \mathrm{msec} / \mathrm{frame})$. The screen of the monitor $\left(12.84^{\circ} \times 9.65^{\circ}\right)$ was masked to subtend $11.4^{\circ}$ horizontally and $8.6^{\circ}$ vertically. A grating pattern that appeared in the center of the screen subtended $4^{\circ} \times 4^{\circ}$ from a viewing distance of $80 \mathrm{~cm}$. The spatial waveforms of the grating were produced with a function generator (Hewlett Packard, 3312A), by means of which the start/stop phase range was adjustable. The edge on both sides of a pattern was always at a zero crossing of the spatial waveform. The spatial frequency of the grating was $0.75,1.5,3$, or $6 \mathrm{cpd}$. These values represent the nominal frequencies of each pattern. Since these stimuli are patches of a fixed spatial extent, other frequencies are physically present-that is, the Fourier spectrum contains a band of spatial frequencies, which, plotted against log frequency, varies with spatial frequency (See the Discussion for the possible effects of the "extraneous" frequency components on RT). The mean retinal illuminance of the screen was varied by placing ND filters (Kodak wratten gelatine filter) in front of an artificial pupil, or by optically superimposing another white screen, which was illuminated and had the same size and approximately the same color as the television screen $(x=0.262, y=0.291$ on the CIE chromaticity coordinates). The mean retinal illuminance was $0.88,8.8$, 88 , or $1,100 \mathrm{Td}$. A black dot $0.2^{\circ}$ in diameter was always presented as a fixation point at the center of the screen. The contrast of the stimulus grating was defined as the difference between the maximum and the minimum luminances of the grating, divided by twice the mean luminance. Threshold measurements were made in the contrast range below 0.4 , where the amplitudes of the harmonic distortion products were less than $5 \%$. RT measurements were made in the same contrast range, except in three of the stimulus condjtions (the highest contrasts for one observer's $0.75 \mathrm{cpd}$ at $0.88 \mathrm{Td}$ condition and for the other observer's 0.75 and $6 \mathrm{cpd}$ at $0.88 \mathrm{Td}$ conditions). For the three conditions, the contrast range of 0.43-0.56 was used, in which the amplitudes of the harmonic distortion products were less than $9 \%$. The stimulus duration was kept constant at $1,052.1 \mathrm{msec}$. The stimulus onset and offset were controlled by a microcomputer and a programmable digital device synchronized with the horizontal driving signal for the television monitor. The contrast of the stimulus grating was varied by the microcomputer with $\mathrm{D} / \mathrm{A}$ converters, $\mathrm{dB}$ attenuators, and analog multipliers. The computer sequenced the stimulus presentations and monitored the observers' responses.

Procedure. An experimental session consisted of threshold measurements and blocks of RT measurements for a given combination of spatial frequency and retinal illuminance. Before the RT measurements, detection threshold was determined by a double random staircase method (Cornsweet, 1962). The procedure has been described in detail elsewhere (Ohtani \& Ejima, 1988). The threshold measurements were repeated at least four times, and the mean of the individual estimates was taken as an average. Immediately after the threshold measurements, the RT experiment began. After 6 min of initial adaptation to a homogeneous screen of a given retinal illuminance, the observer was given self-initiated trials, each of which was started by the observer's press of a microswitch. In each trial, a stimulus grating was presented after a 1-sec warning buzzer had been followed by a randomly variable foreperiod of $1,000-1,500 \mathrm{msec}$. The observer responded by releasing the microswitch as fast as possible after he detected a stimulus. RT was measured as the time between the stimulus onset and the release of the microswitch, with a precision of $1 \mathrm{msec}$. RTs longer than $1,000 \mathrm{msec}$ or shorter than $100 \mathrm{msec}$ were treated as misses and false alarms, respectively, and were excluded from data analysis.

Each experimental block consisted of $10 \mathrm{RT}$ measurements for each of eight contrast levels, resulting in a total of $\mathbf{8 0}$ trials. The eight contrast values were $3,4,6,8,10,12,16$, and $20 \mathrm{~dB}$ above threshold contrast, except in the $0.75 \mathrm{cpd}$ at $1,100 \mathrm{Td}$ condition for one observer, for which the highest two contrasts were 14 and $18 \mathrm{~dB}$ instead of 16 and $20 \mathrm{~dB}$. In each block, the different contrasts were presented in random order, and median RTs were determined for the eight contrast values. A total of 10 blocks were run consecutively with a break of several minutes between blocks. Spatial frequency and retinal illuminance were varied between sessions. The authors served as observers; both were emmetropic. The observer viewed the stimulus with his right eye through an artificial pupil $2 \mathrm{~mm}$ in diameter.

\section{Results}

Figure 1 shows RT as a function of stimulus contrast for the two observers; the left column is for Observer Y.E., the right for Observer Y.O. The four panels in each column represent the results for the different spatial frequencies indicated at the right. In each panel, the ordinate denotes RT and the abscissa denotes relative contrast scaled in decibels. Different symbols represent the results for different retinal illuminances; circles for $0.88 \mathrm{Td}$, tri-

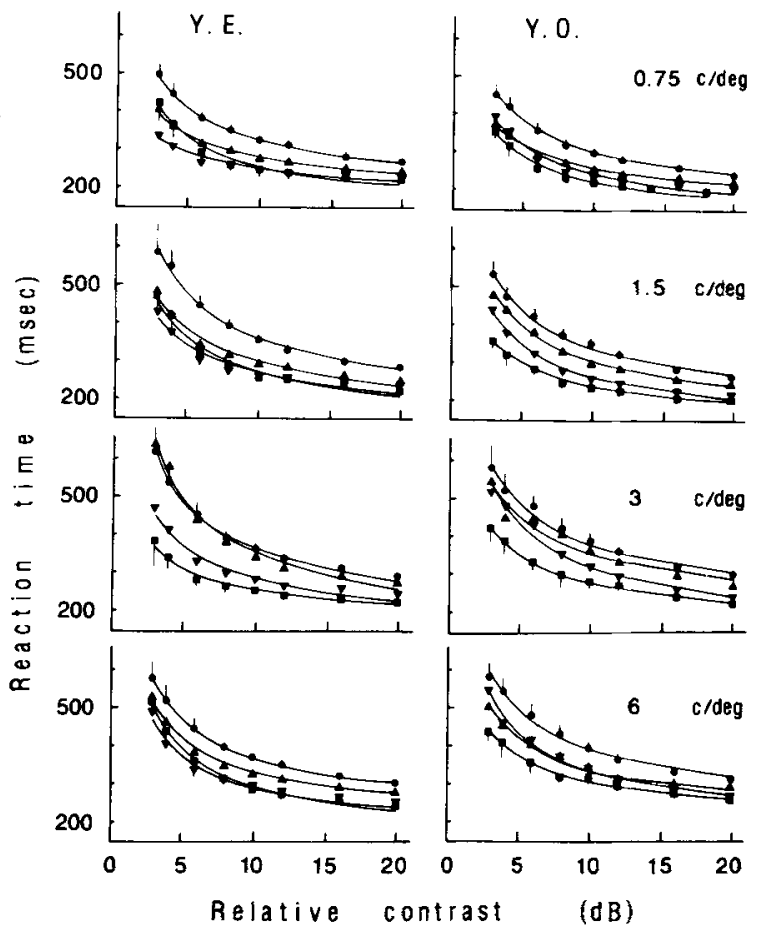

Figure 1. Reaction time as a function of contrast for four spatial frequencies at four retinal illuminances, with the left column for Y.E. and the right for Y.O. The four panels in each column represent the results for the different spatial frequencies indicated at the right. Different symbols denote the results for different retinal illuminances: circles for $0.88 \mathrm{Td}$, triangles for $8.8 \mathrm{Td}$, inverted triangles for $88 \mathrm{Td}$, and squares for 1,100 Td. Each datum point is the average of 10 median RTs, and the vertical bars represent $1 S D$ of the median RTs. Standard deviations are shown only for the data of the lowest and highest retinal illuminances, for clarity of the figure. Solid curves are the best-fitting functions obtained with Equation 5 . 
angles for $8.8 \mathrm{Td}$, inverted triangles for $88 \mathrm{Td}$, and squares for 1,100 Td. Each data point shows the average of 10 median RTs. ${ }^{1}$ Standard deviations of the median RTs are indicated by the vertical bars at the individual data points and are shown only for the lowest and highest retinal illuminances, for clarity of the figure. For all the conditions of spatial frequency and retinal illuminance employed here, RT decreases monotonically with increasing grating contrast. Solid curves through the data points represent the theoretical functions of the two-stage model mentioned later.

\section{ESTIMATION OF THE VISUAL LATENCY CONTRAST FUNCTION}

It has been shown that temporal properties of the visual system at threshold level can be described well with a linear filter model in which it is assumed that the visual system consists of a set of spatiotemporal linear filters (Bergen \& Wilson, 1985; Gorea \& Tyler, 1986; Watson, 1979, 1986; Watson \& Nachmias, 1977). If one assumes that RT is determined by the sensory signal near threshold level, ${ }^{2}$ the linear filter model may be applied to obtain the time course of the sensory signal in the vicinity of the critical level, and the sensory processing delay (the visual latency) involved in RT may thereby be estimated.

It is well known that once the impulse response of the linear filter is obtained, the filter's response to any temporal stimulus can be derived by convolving the impulse response with the stimulus's temporal waveform. The gradual, rather than instantaneous, increase in the filter's response amplitude gives rise to a delay between the time of the start of the filter's response and the time at which threshold level is reached. This delay constitutes one component of the visual latency. The visual latency may involve another component, which may be ascribed to the response latency at the peripheral process and the transmission time of the visual signal from the peripheral to the central process. This component constitutes the ab-

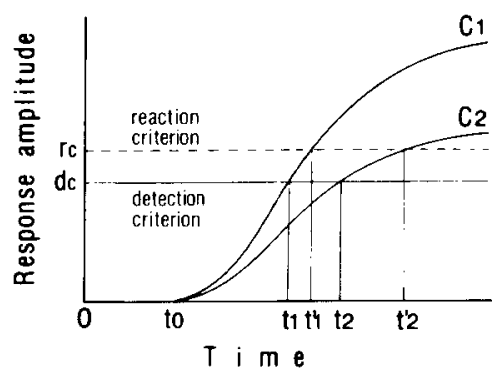

Figure 2. Procedure for estimation of the visual and response latency. The two curves represent the linear filter's response for gratings of contrasts $\mathrm{Cl}$ and $\mathrm{C2}$. The horizontal solid line denotes detection threshold level, $d_{c}$. The intersections of the line with the two curves define the visual latencies of $t_{1}$ and $t_{2}$ for the two contrasts. The dashed horizontal line denotes the response criterion, $r_{c}$, set at a level higher than detection threshold. The intersections of the line with the two curves define the response latencies of $t_{1}^{\prime}$ and $t_{2}^{\prime}$ for the two contrasts.

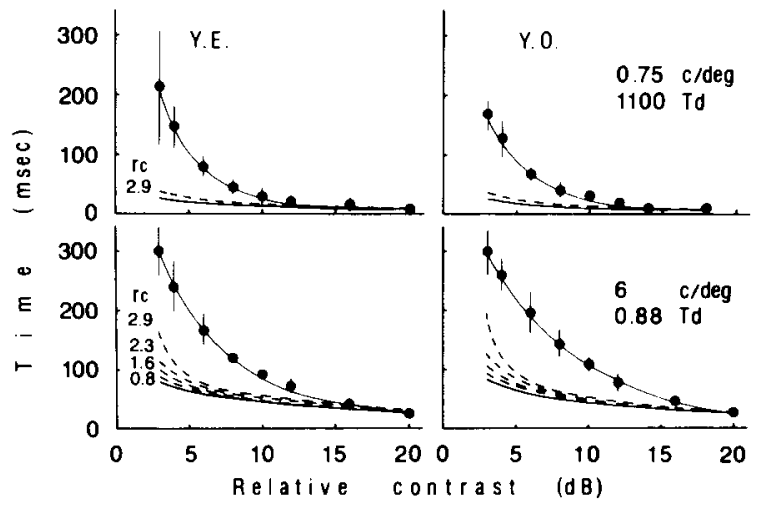

Figure 3. Theoretical visual latency (VL') and response latency (RL') as a function of contrast for the 0.75 cpd grating at 1,100 Td and the 6 cpd grating at $0.88 \mathrm{Td}$; the left column is for Y.E. and the right for Y.O. Solid thick curves represent the visual latency functions. Dashed curves represent the response latency functions with $r_{c}$ set at different levels higher than detection threshold. The four numbers at the left indicate the $r_{c}$ levels expressed in units of dB. For the $0.75 \mathrm{cpd}$ at $1,100 \mathrm{Td}$, the response latency functions with the $r_{c}$ levels of 0.8-2.3 dB are omitted for clarity's sake. For comparison, RT data (connected by smooth, thin curves) are also plotted displaced, so as to meet the visual latency functions at the highest contrast. Vertical bars at the individual data points indicate $\pm 1 S D$.

solute latency of the filter's response. The two curves in Figure 2 schematically illustrate the filter's responses for suprathreshold gratings with contrasts $\mathrm{C} 1$ and $\mathrm{C} 2$. In the figure, $t_{0}$ denotes the absolute latency of the filter's response, and the solid horizontal line represents the detection threshold level, $d_{c}$. The intersections of the line with the two curves define the visual latencies (VLs; $t_{1}$ and $t_{2}$ ) for the two contrasts. Since $t_{0}$ is not specified by threshold measurements, it is not the value of VL but that of $\mathrm{VL}^{\prime}$, defined as the difference between $\mathrm{VL}$ and $t_{0}$, that can be obtained from the impulse responses, given the threshold data.

Using the impulse responses obtained in the previous study (Ohtani \& Ejima, 1988), we derived the filter's responses for suprathreshold gratings and determined the VL' contrast functions. ${ }^{3}$ Specifically, we first convolved the impulse response with a 1,052.1-msec temporal rectangular pulse and divided the filter's response as a function of time by the peak amplitude of the response. Next, the filter's response for a suprathreshold grating as a function of time was obtained through multiplying the normalized filter's response by the suprathreshold-tothreshold contrast ratio. With this procedure, the threshold level $d_{c}$ corresponds to the filter's response amplitude, with the value of 1 . Then, we determined the visual latency for the suprathreshold grating at which the filter's response first exceeded the $d_{c}$ level.

The solid thick curves in Figure 3 are two examples of the VL' contrast functions estimated in the same contrast range as that used in the RT measurements (the left column for Y.E., and the right for Y.O.): one for the 0.75 -cpd grating at $1,100 \mathrm{Td}$, and the other for the 6-cpd 
grating at $0.88 \mathrm{Td}$. The two conditions shown here are those in which VL' shows the smallest and the largest contrast dependence of all the conditions of spatial frequency and retinal illuminance. As the stimulus contrast is decreased from 20 to $3 \mathrm{~dB}$ above detection threshold, VL' for the 0.75 -cpd grating at $1,100 \mathrm{Td}$ increases by $15 \mathrm{msec}$. For the 6-cpd grating at $0.88 \mathrm{Td}$, it increases by $50-60 \mathrm{msec}$. The present variation of $\mathrm{VL}^{\prime}$ with stimulus contrast, which falls between 15 and $60 \mathrm{msec}$, depending on spatial frequency and retinal illuminance, is generally in accord with that found in previous studies, in which the visual latencies were empirically determined on the basis of the Pulfrich effect (Alpern, 1968; Brauner \& Lit, 1976; Williams \& Lit, 1983), the Hess effect (Williams \& Lit, 1983; Wilson \& Anstis, 1969), the apparent simultaneity of the onset or offset of two stimuli (Arden \& Weale, 1954; Roufs, 1963, 1974; Lewis, Dunlap, \& Matteson, 1972), and the apparent synchrony of rotation of two disks (Prestrude, 1971; Prestrude \& Baker, 1968, 1971).

For comparison of the magnitudes of contrast dependence, the corresponding RT data are also plotted in Figure 3 (displaced so as to meet the visual latency functions at the highest contrast). As is evident from the figure, the contrast dependence of RT $(200-270 \mathrm{msec})$ is much larger than that of the visual latency $(15-60 \mathrm{msec})$. Roufs (1974) has reported a similar divergence between the empirical RT intensity functions for foveal lights and the theoretical visual latency functions.

\section{ANALYSIS OF DISCREPANCY BETWEEN RT AND VISUAL LATENCY}

\section{Criterion Difference Model}

A qualitative explanation of the discrepancy between RT and the visual latency has been advanced by the present authors (Ejima \& Ohtani, 1987). On the basis of Grice's decision-theory approach, they suggested that the discrepancy might be explained with a model in which a difference in response criterion for detection and reaction is assumed. According to this criterion difference model, RT is described in the same manner as in Equation 1 , and the response criterion, $r_{c}$, is set at a higher level than detection threshold. With $r_{c}$ set at the level indicated by the dashed horizontal line in Figure 2, response latencies (RLs) for the two contrasts $C 1$ and $C 2$ are defined as $t_{1}^{\prime}$ and $t_{2}^{\prime}$, respectively. For the same amount of change in stimulus contrast, the difference between the response latencies, $t_{2}^{\prime}-t_{1}^{\prime}$, is larger than that between the visual latencies, $t_{2}-t_{1}$. Response delay due to the criterion elevation is expressed as $\left(t_{2}^{\prime}-t_{1}^{\prime}\right)-\left(t_{2}-t_{1}\right)$. If the criterion difference model is correct, the response delay will reconcile the present divergence between the RT and visual latency functions.

In order to evaluate the criterion difference model, we calculated the theoretical response latency contrast functions at different $r_{c}$ levels (response latency, $\mathrm{RL}^{\prime}$, is defined as $R L-t_{0}$ ). The family of the dashed curves in
Figure 3 represents the $R L^{\prime}$ functions. The values of $r_{c}$ are given in decibels at the left. It is shown that, as the response criterion is elevated, the contrast dependence of the response latency functions increases, reconciling the divergence between the RT and visual latency functions. However, the magnitude of the contrast dependence of the response latency functions is still much smaller than that of the RT functions. For the 0.75-cpd grating at $1,100 \mathrm{Td}$, the variation of $\mathrm{RL}^{\prime}$ with stimulus contrast is $25 \mathrm{msec}$, even when $r_{c}$ is raised to as high as $2.9 \mathrm{~dB}$ above threshold contrast. For the 6-cpd grating at $0.88 \mathrm{Td}$, the variation of RL' with this $r_{c}$ level is $140-170 \mathrm{msec}$. Thus, the criterion difference model cannot entirely reconcile the discrepancy between the RT and the visual latency functions in its present form.

\section{Functional Relation Between RT and}

\section{the Time Course of the Linear Filter's Response}

In the linear filter model (including the criterion difference model), with which the present visual latency and response latency functions were derived, it is assumed that behavioral responses are initiated when the filter's response exceeds a threshold. This type of model, which may be called a level detector model, has been successfully useful in the explanation of the temporal properties of the visual system at threshold level, but it failed to reconcile the discrepancy between $R T$ and the visual latency. The failure of the linear filter model to explain the RT functions suggests that the RT process may involve another type of mechanism, which acts on some aspect of the filter's response other than amplitude. One possible formulation to involve such a mechanism might be the following:

$$
\begin{aligned}
\mathrm{RT}= & \min \left[t ; R(t)>d_{\mathrm{c}}\right] \\
& +G\left[R^{\prime}\left(t_{\min }\right), R^{\prime \prime}\left(t_{\min }\right), \ldots, R^{n}\left(t_{\min }\right)\right],
\end{aligned}
$$

where $G$ represents a function, and $R^{\prime}\left(t_{\min }\right), R^{\prime \prime}\left(t_{\min }\right), \ldots$, $R^{n}\left(t_{\min }\right)$ are the first, second, .., $n$th order differential coefficients of the filter's response at the time of $t_{\min }$, respectively.

We explored a simple form of Equation 2 that can reconcile the discrepancy between $\mathrm{RT}$ and the visual latency, thereby explaining the RT functions. Burbeck and Luce have recently demonstrated the contribution to auditory RT of a mechanism that operates as a differentiator in sensory information processing (Burbeck, 1985; Burbeck \& Luce, 1982; Luce, 1986). If the contribution of such a differentiator to the RT process is incorporated without regard for the higher order differential terms, Equation 2 becomes reduced to

$$
\mathrm{RT}=\min \left[\mathrm{t} ; R(t) \geq d_{\mathrm{c}}\right]+G\left[R^{\prime}\left(t_{\min }\right)\right]+K .
$$

Preliminary analyses concerning the functional form of $G$ revealed that the difference between RT and VL' could be characterized in terms of a decreasing linear function of the slope of the filter's response at threshold level [i.e., $\left.R^{\prime}\left(t_{\min }\right)\right]$ on double logarithmic coordinates. This sug- 
gested that the discrepancy between RT and the visual latency might be described by a power function of the slope of the filter's response at threshold level. Therefore, we adopted the following equation:

$$
G\left[R^{\prime}\left(t_{\min }\right)\right]=a R^{\prime}\left(t_{\min }\right)^{-b},
$$

where $a$ and $b$ are constants. Then, Equation 3 becomes

$$
\begin{aligned}
\mathrm{RT} & =\min \left[t ; R(t) \geq d_{c}\right]+a R^{\prime}\left(t_{\min }\right)^{-b}+K \\
& =\left\{\min \left[t ; R(t) \geq d_{c}\right]-t_{0}\right\}+a R^{\prime}\left(t_{\min }\right)^{-b}+R T_{0},
\end{aligned}
$$

with which we fitted the RT functions. The value of $\left\{\min \left[t ; R(t) \geq d_{c}\right]-t_{0}\right\}$ is the same as the $\mathrm{VL}^{\prime}$ estimated in the previous section, and $\mathrm{RT}_{0}$ is equal to $t_{0}+K . R^{\prime}\left(t_{\min }\right)$ for each contrast (in units of threshold unit/millisecond) was calculated numerically from the filter's response for the contrast. The optimal values of $a, b$, and $\mathrm{RT}_{0}$ were estimated concurrently, so as to minimize the sum of the squares of the difference between the empirical data and the model's prediction.

Table 1

\begin{tabular}{|c|c|c|c|c|c|}
\hline Illuminance & $\begin{array}{c}\text { Spatial } \\
\text { Frequency }\end{array}$ & $a$ & $b$ & $\mathrm{RT}_{0}$ & $r$ \\
\hline \multicolumn{6}{|c|}{ Observer Y.E. } \\
\hline \multirow[t]{4}{*}{0.88} & 0.75 & 9 & 0.75 & 171 & .999 \\
\hline & 1.5 & 10 & 0.79 & 170 & .995 \\
\hline & 3 & 10 & 0.79 & 176 & .999 \\
\hline & 6 & 10 & 0.77 & 209 & 1.000 \\
\hline \multirow[t]{4}{*}{8.8} & 0.75 & 10 & 0.73 & 154 & .990 \\
\hline & 1.5 & 10 & 0.80 & 136 & .988 \\
\hline & 3 & 11 & 0.85 & 134 & .990 \\
\hline & 6 & 9 & 0.76 & 177 & .994 \\
\hline \multirow[t]{4}{*}{88} & 0.75 & 12 & 0.69 & 153 & .971 \\
\hline & 1.5 & 12 & 0.83 & 133 & .976 \\
\hline & 3 & 13 & 0.81 & 138 & .981 \\
\hline & 6 & 12 & 0.80 & 167 & .985 \\
\hline \multirow[t]{4}{*}{1,100} & 0.75 & 13 & 1.00 & 139 & .981 \\
\hline & 1.5 & 14 & 0.93 & 125 & .986 \\
\hline & 3 & 12 & 0.76 & 147 & .985 \\
\hline & 6 & 14 & 0.86 & 146 & .992 \\
\hline \multicolumn{6}{|c|}{ Observer Y.O. } \\
\hline \multirow[t]{4}{*}{0.88} & 0.75 & 9 & 0.74 & 154 & .998 \\
\hline & 1.5 & 9 & 0.74 & 185 & .996 \\
\hline & 3 & 9 & 0.72 & 212 & .992 \\
\hline & 6 & 11 & 0.72 & 227 & .994 \\
\hline \multirow[t]{4}{*}{8.8} & 0.75 & 9 & 0.73 & 142 & .990 \\
\hline & 1.5 & 10 & 0.79 & 151 & .997 \\
\hline & 3 & 10 & 0.73 & 194 & .976 \\
\hline & 6 & 8 & 0.72 & 203 & .998 \\
\hline \multirow[t]{4}{*}{88} & 0.75 & 14 & 0.79 & 117 & .979 \\
\hline & 1.5 & 13 & 0.81 & 123 & .993 \\
\hline & 3 & 16 & 0.77 & 146 & .990 \\
\hline & 6 & 13 & 0.80 & 195 & .995 \\
\hline \multirow[t]{4}{*}{1,100} & 0.75 & 15 & 0.86 & 121 & .988 \\
\hline & 1.5 & 14 & 0.79 & 139 & .993 \\
\hline & 3 & 15 & 0.75 & 149 & .999 \\
\hline & 6 & 11 & 0.73 & 199 & .995 \\
\hline
\end{tabular}

Parameter Values Estimated to Fit the RT Functions, Using Equation 5

Note-Illuminance is given in trolands. Spatial frequency is given in cycles per degree (cpd). $r=$ Pearson product-moment correlation.
The best-fitting functions are shown as solid curves in Figure 1. The quality of fit of the estimated functions to the data is excellent, implying that no other higher order differential terms in Equation 2 are required to describe the RT functions. The optimal parameter values with the Pearson product-moment correlation coefficients are listed in Table 1. Of the two parameters related to the differentiator, the exponent parameter, $b$, has similar values across the experimental conditions and observers. The average of the exponents is $0.81(S D=0.076)$ for Y.E., and 0.76 $(S D=0.041)$ for Y.O. This result indicates that the slope-dependent component of RT is characterized by a common exponent of about 0.8 , independent of spatial frequency and retinal illuminance. The scaling parameter, $a$, has similar values for different spatial frequencies, but not for different retinal illuminances; it is somewhat larger at the higher retinal illuminances $(88$ and $1,100 \mathrm{Td} ; 13$ or 14) than at the lower retinal illuminances $(0.88$ and $8.8 \mathrm{Td}$; about 9). $\mathrm{RT}_{0}$, which represents asymptotic $\mathrm{RT}$, depends on spatial frequency and retinal illuminance. As spatial frequency is increased and/or retinal illuminance is decreased, $\mathrm{RT}_{0}$ becomes larger, although the magnitude of its variation with spatial frequency is smaller for Y.E. than for Y.O. Similar effects of spatial frequency and adaptation level on the asymptotic RT have been previously reported (Bartlett \& MacLeod, 1954; Ejima \& Ohtani, 1987; Harwerth \& Levi, 1978). The variation of $\mathrm{RT}_{0}$ with these stimulus parameters may be ascribed to that of the absolute latency of the filter's response $\left(t_{0}\right)$, which involves the response latency at the peripheral visual process and/or the transmission time of the visual signal.

\section{DISCUSSION}

Models of the RT process have been developed which concern how reaction time depends on task requirements (e.g., simple reaction, go/no go reaction, and choice reaction, including identification) and on (stochastic) variation of both the amplitude of the sensory signal and the response criterion (Grice, 1968; Luce \& Green, 1972; McGill, 1963). In these studies, the sensory signal has been represented as a train of neural pulses, and the decision rules have been stated in terms of aspects of the pulse train, such as the cumulative number of pulses (Grice, 1968; McGill, 1963) or the interarrival time between successive pulses (Luce \& Green, 1972). Input to the decision process has been related to stimulus parameters in a simple manner. For instance, Luce and Green (1972) have assumed that immediately after stimulus onset, the intensity parameter takes some value (depending on the stimulus intensity) and changes the density of the input pulses to the decision process. Grice (1968) has assumed that stimulus intensity controls the slope of the linearly increasing function of the cumulative number of input pulses after stimulus onset. The functional relation between the input to the decision process and the early sensory signal has not been a matter of strong 
concern. The present study sheds light on this functional relation.

\section{Derivation of the Visual Latency Function} on the Basis of a Single-Filter Assumption

It has been shown that RT intensity functions do not conform straightforwardly to visual latency intensity functions. To analyze the difference between the RT and the visual latency functions, we determined the RT contrast functions for sinusoidal gratings empirically, and we derived the visual latency contrast functions from threshold data theoretically by using a linear filter model. The theoretical derivation was based on the assumption that the time course of the sensory signal for temporal rectangular pulses, for a given combination of spatial frequency and adaptation level, may be characterized well by the temporal response of a single linear filter.

The single-filter assumption may be oversimplified in view of the physical property of the present stimuli and the complexity of the spatiotemporal mechanisms of the human visual system. First, since the present stimuli are patches of sinusoidal gratings of a fixed spatial extent, their Fourier amplitude spectra contain "extraneous" frequency components whose amplitudes increase with increases in stimulus contrast, as well as the fundamental ones. At lower contrasts, the responses to the extraneous frequency components presumably may be below threshold, but at higher contrasts, some of them may exceed the threshold level. Thus, the single-filter assumption may not strictly hold over the whole range of contrasts employed here. Second, it has been shown that the temporal processing of a flickering pattern, as revealed by the discriminability, masking, and threshold temporal frequency functions, is characterized not by a single channel, but by multiple channels (Lehky, 1985; Mandler \& Makous, 1984; Ohtani \& Ejima, 1988).

By using the multiple-, rather than the single-filter, assumption, one may develop a model with which the discrepancy between the RT and the visual latency functions can be analyzed. But analysis based on the multiple-filter assumption would require knowledge not only of the spatiotemporal tuning of the mechanisms, but also of their latencies and of how their responses combine. Unfortunately, the existing literature does not provide data sufficient to develop a quantitative model. On the other hand, there are indications that models based on the single-filter assumption can explain some aspects of the suprathreshold temporal properties of the visual system (Georgeson, 1987; Roufs, 1974). Thus, it seems worthwhile at present to explore the ramifications of the single-filter assumption as a first approximation of the description of the RT process.

\section{A Two-Stage Model of the RT Process}

The present RT contrast functions may be described successfully in terms of the visual latency and the slope of the linear filter's response at threshold level, both of which were derived from the time course of the linear filter's response. This suggests that the RT process may involve two processing stages-a level detector and a differentiator-which operate on different aspects of the filter's response. The level detector, which monitors the amplitude of the filter's response, determines the visual latency. The differentiator, which monitors the temporal change of the filter's response, gives rise to the slopedependent component of RT. Note here that the differentiator was required to describe the RT functions for all the experimental conditions, irrespective of the temporal response property of the linear filter. This suggests that the linear filter and the differentiator represent different stages in the RT process, the latter of which is a higher order mechanism that operates on the filter's response.

Figure 4 schematically illustrates one plausible model that illustrates the present formulation conceptually. The RT process consists of multiple subprocesses, which are represented by boxes and circles connected by solid lines. The first element is a linear filter (LF), which translates the incoming signal into neural terms. The circle in the parentheses, which means that the filter's response is perturbed by additive noise, is added in order to make this model consistent with a model of the detection process, but this element is not essential. The linear filter is followed by an analog threshold device, which allows the input signal to pass through only when it exceeds threshold level, $d_{c}$. The processing stage with the collection of elements up to and including the analog threshold device consists of the level detector. The output signal of the analog threshold device passes through a nonlinear differentiator, which monitors the temporal change in the input signal. The present results suggest that the nonlinear property of the differentiator may be described by a power function, but this does not eliminate the possibility of success-

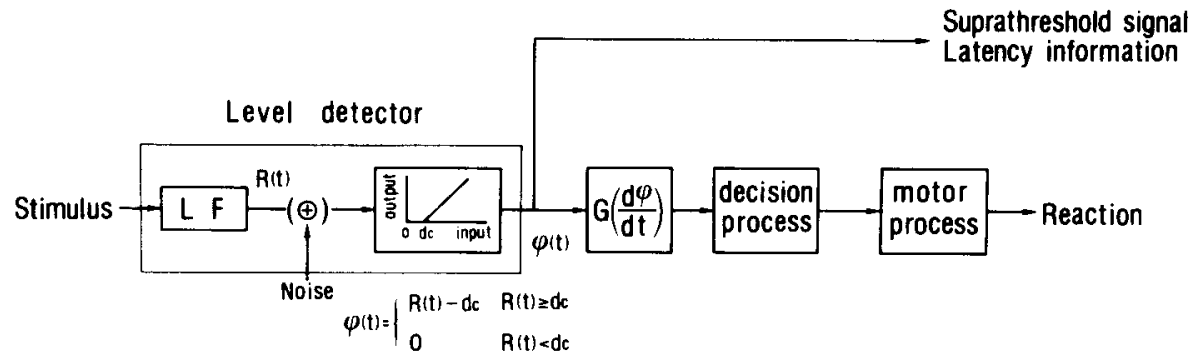

Figure 4. A two-stage model of the RT process. LF = linear filter. 
ful formulation with other functional forms. The differentiator's output is then fed into the decision process, which accumulates input signal and activates the motor process whenever the accumulated signal exceeds a criterion level. Thus, the contrast-dependent component of RT is a sum of the latency of the level detector (defined as the interval between stimulus onset and the time at which the filter's response exceeds detection threshold) and the decision latency (defined as the interval between the time at which the analog threshold device first yields nonzero response and that at which the motor process is activated). The latency of the level detector corresponds to the visual latency estimated in the present study. The rightward arrow branching off from the analog threshold device output indicates that the suprathreshold signal and latency information of the level detector are available for tasks other than simple reaction (e.g., stimulus detection, simultaneity judgment).

The RT functions and their divergence from the visual latency functions can be explained over a wide range of contrasts with the present model. For high-contrast stimuli, the decision latency would be small, since these stimuli would yield the filter's responses with steeper slope at threshold level. The decision process would respond as soon as the level detector is activated. This would lead to correspondence between the RT and visual latency functions. With decreases in contrast, the decision latency would be increased, since the slope of the filter's response at threshold level decreases with contrast. This would produce an increase in RT greater than that in the visual latency, leading to progressive divergence between the RT and visual latency functions. At the lower extremity, where the slope of the filter's response at threshold level is reduced to zero, RT becomes infinitely long theoretically (Equation 5). By definition, however, any detectable stimulus yields a nonzero slope of the filter's response at threshold level, and consequently yields a finite RT. Even with a nonzero slope, a weak stimulus may be missed in an RT task if the total processing delay exceeds the upper limit of RT measurement.

A model of the RT process relevant to the present one is the two-detector model for auditory RT proposed by Burbeck and Luce (Burbeck, 1985; Burbeck \& Luce, 1982; Luce, 1986). The present level detector and differentiator are similar to the level and change detectors in their model, but the underlying ideas are different in some respects. First, Luce (1986) supposed that the change detector in vision might correspond to the transient channels. On the other hand, it is likely that the differentiator in the present model operates at a different processing stage from that at which the transient channels do. Second, the Burbeck and Luce model is a race model that involves the two types of detector, such that whichever detector detects a stimulus first will trigger a response. By contrast, from the consideration of the processing stages at which the level detector and the differentiator operate, we suppose that the two contribute to RT not alternatively, but concurrently in sequence.

\section{Power Law for the RT Intensity Function}

It is well known that a power law (Pieron's law) holds between the reducible component of RT and stimulus intensity (Mansfield, 1973; Vaughan et al., 1966). The present study demonstrates that the relation between the reducible component of RT with the visual latency excluded and the slope of the filter's response at threshold level can be described by a power law. It is interesting that a similar power law holds for the RT sensory slope relation as well as the RT intensity relation. Considering the fact that the slope of the filter's response estimated in the present study is approximately linearly related to stimulus contrast, the similarity is of no surprise. It is to be emphasized here that the present model provides a view of the underlying process mediating the RT intensity relation through the sensory system. Our understanding of the RT process will be improved by the examination of the functionally significant aspect of the sensory signal as well as that of the stimulus parameter.

\section{REFERENCES}

AlPern, M. (1968). A note on visual latency. Psychological Review, 75, 260-264.

ARDEN, G. B., \& Weale, R. A. (1954). Variations of the latent period of vision. Proceedings of the Royal Society of London, B142, 258-267.

Bartlett, N. R., \& MACLEOD, S. (1954). Effect of flash and field luminance upon human reaction time. Journal of the Optical Society of America, 44, 306-311.

BARTLETT, N. R., STicht, T. G. (1968). Effects of wavelength and retinal locus on the reaction time to onset and offset stimulation. Joumal of Experimental Psychology, 78, 699-701.

Bergen, J. R., \& WiLson, H. R. (1985). Prediction of flicker sensitivities from temporal three-pulse data. Vision Research, 25, 577-582.

Brauner, J. D., \& LiT, A. (1976). The Pulfrich effect, simple reaction time, and intensity discrimination. American Joumal of Psychol ogy, 89, $105-114$.

Bruder, G. E., \& Kietzman, M. L. (1973). Visual temporal integration for threshold, signal detectability, and reaction time measures. Perception \& Psychophysics, 13, 293-300.

BURBECK, S. L. (1985). A physiologically motivated model for change detection for audition. Journal of Mathematical Psychology, 29, 106-121.

Burbeck, S. L., \& Luce, R. D. (1982). Evidence from auditory simple reaction times for both change and level detectors. Perception \& Psychophysics, 32, 117-133.

Burkhardt, D. A., Gottesman, J., \& KeEnan, R. M. (1987). Sensory latency and reaction time: Dependence on contrast polarity and early linearity in human vision. Journal of the Optical Society of America, 4, 530-539.

CORNSWEET, T. N. (1962). The staircase-method in psychophysics. American Joumal of Psychology, 75, 485-491.

EJima, Y., \& OHTANI, Y. (1987). Simple reaction time to sinusoidal grating and perceptual integration time: Contributions of perceptual and response processes. Vision Research, 27, 269-276.

GEORGESON, M. A. (1987). Temporal properties of spatial contrast vision. Vision Research, 27, 765-780.

GoreA, A., \& TYler, C. W. (1986). New look at Bloch's law for contrast. Journal of the Optical Society of America, A3, 52-61.

Grice, G. R. (1968). Stimulus intensity and response evocation. Psychological Review, 75, 359-373.

GrossberG, M. (1968). The latency of response in relation to Bloch's law at threshold. Perception \& Psychophysics, 4, 229-232.

HARWERTH, R. S., \& LEVI, D. M. (1978). Reaction time as a measure of suprathreshold grating detection. Vision Research, 18, 1579-1586.

KiETZMAN, M. L., \& GillaM, B. J. (1972). Visual temporal integra- 
tion and simple reaction time. Perception \& Psychophysics, 11, 333-340.

LEHKY, S. R. (1985). Temporal properties of visual channels measured by masking. Journal of the Optical Society of America, A2, 1260-1272.

Lewis, J. H., Dunlap, W. P., \& Matteson, H. H. (1972). Perceptual latency as a function of stimulus onset and offset and retinal location. Vision Research, 12, 1725-1731.

LuCE, R. D. (1986). Response times. New York: Oxford University Press.

LuCE, R. D., \& Green, D. M. (1972). A neural timing theory for response times and the psychophysics of intensity. Psychological Review, 79, 14-57.

Mandler, M. B., \& Makous, W. (1984). A three channel model of temporal frequency perception. Vision Research, 24, 1881-1887.

MANSFiEld, R. J. W. (1973). Latency functions in human vision. Vision Research, 13, 2219-2234.

McGill, W. J. (1963). Stochastic latency mechanisms. In R. D. Luce, R. R. Bush, \& E. Galanter (Eds.), Handbook of Mathematical Psychology (Vol. 1, pp. 309-360). New York: Wiley.

Nissen, M. J., \& Pokorny, J. (1977). Wavelength effects on simple reaction time. Perception \& Psychophysics, 22, 457-462.

Ohtani, Y., \& EJIMA, Y. (1988). Relation between flicker and twopulse sensitivities for sinusoidal gratings. Vision Research, 28, 145-156.

Pollack, J. D. (1968). Reaction time to different wavelengths at various luminances. Perception \& Psychophysics, 3, 17-24.

Prestrude, A. M. (1971). Visual latencies at photopic levels of retinal illuminance. Vision Research, 11, 351-361.

Prestrude, A. M., \& BaKer, H. D. (1968). New method of measuring visual-perceptual latency differences. Perception \& Psychophysics, 4, 152-154.

Prestrude, A. M., \& BAKER, H. D. (1971). Light adaptation and visual latency. Vision Research, 11, 363-369.

RaAB, D., Fehrer, E. (1962). The effect of stimulus duration and luminance on visual reaction time. Journal of Experimental Psychology, 64, 326-327.

RAINS, J. D. (1963). Signal luminance and position effects in human reaction time. Vision Research, 3, 239-251.

Roufs, J. A. J. (1963). Perception lag as a function of stimulus luminance. Vision Research, 3, 81-91.

Rours, J. A. J. (1974). Dynamic properties of vision: V. Vision Research, 14, 853-869.

UENO, T. (1976). Luminance-duration relation in reaction time to spectral stimuli. Vision Research, 16, 721-725.

UENO, T. (1977). Reaction time as a measure of temporal summation at suprathreshold levels. Vision Research, 17, 227-232.
Ueno, T. (1978). Temporal summation in human vision: Simple reaction time measurements. Perception \& Psychophysics, 23, 43-50.

Ueno, T., PoKorny, J., \& Smith, V. C. (1985). Reaction times to chromatic stimuli. Vision Research, 25, 1623-1627.

Vaughan, H. G., Costa, L. D., \& Gilden, L. (1966). The functional relation of visual evoked response and reaction time to stimulus intensity. Vision Research, 6, 645-656.

Watson, A. B. (1979). Probability summation over time. Vision Research, 19, 515-522.

Watson, A. B. (1986). Temporal sensitivity. In K. R. Boff, L. Kaufman, \& J. P. Thomas (Eds.), Handbook of perception and human performance: Vol. 1. Sensory processes and perception (chap. 6, pp. 6.1-6.43). New York: Wiley.

Watson, A. B., \& Nachmias, J. (1977). Patterns of temporal interaction in the detection of gratings. Vision Research, 17, 893-902.

Williams, J. M., \& LIT, A., (1983). Luminance-dependent visual latency for the Hess effect, the Pulfrich effect, and simple reaction time. Vision Research, 23, 171-179.

WiLson, J. A., \& ANSTis, S. M. (1969). Visual delay as a function of luminance. American Journal of Psychology, 82, 350-358.

\section{NOTES}

1. After exclusion of false alarms and misses, 7-10 RT measurements were available in calculating each of the total of 2,560 median RTs except two, for which 5 and 6 RT measurements were available. For each combination of spatial frequency and retinal illuminance, the proportion of false alarms was less than $5 \%$ and that of misses was less than $1.5 \%$.

2. The assumption is supported by the results of the previous studies on the effect of stimulus duration on RT to flashes of light (Kietzman \& Gillam, 1972; Mansfield, 1973; Raab \& Fehrer, 1962; Ueno, 1977). These studies have shown that decreases in RT with increases in stimulus duration occur only for shorter durations of several tens of milliseconds at most. This suggests that reactions are triggered by the early portion of the sensory signal, presumably near threshold level, rather than by the peak or the later portion, which varies significantly with stimulus duration.

3. For the 0.75 and .15 cpd gratings at 88 and 1,100 Td, the impulse responses used are those which have been modified on the basis of the duplicity hypothesis to fit the two-pulse sensitivity data (see Ohtani \& Ejima, 1988, Table 2).

(Manuscript received June 15, 1988; revision accepted for publication January 25, 1989.) 